\title{
INFLUÊNCIA DAS CARACTERÍSTICAS DOS PAÍSES NA DISSEMINAÇÃO DA COVID-19
}

\author{
INFLUENCE OF COUNTRY CHARACTERISTICS ON THE DISSEMINATION OF COVID-19
}

\section{DYENIFFER PACKER EIGENSTUHLER}

Mestranda do PPG em Ciências Contábeis e Administração

Universidade Comunitária da Região de Chapecó - Unochapecó

Orcid: https://orcid.org/0000-0001-8930-1720 / E-mail: dyeniffer@unochapeco.edu.br Rua Princesa Isabel, 1980-E, Jardim América, Chapecó-SC, CEP: 89803-442

FRANCIELI PACASSA

Mestranda do PPG em Ciências Contábeis e Administração Universidade Comunitária da Região de Chapecó - Unochapecó

Orcid: https://orcid.org/0000-0002-4551-3112 / E-mail: francielipacassa@unochapeco.edu.br

\section{SILVANA DALMUTT KRUGER}

Doutora em Contabilidade - Universidade Federal de Santa Catarina (UFSC)

Professora do PPG em Ciências Contábeis e Administração - Unochapecó

Orcid: https://orcid.org/0000-0002-3353-4100 / E-mail: silvanak@unochapeco.edu.br

\section{SADY MAZZIONI}

Doutor em Ciências Contábeis e Administração - Universidade Regional de Blumenau (FURB)

Professor do PPG em Ciências Contábeis e Administração - Unochapecó

Orcid: https://orcid.org/0000-0002-8976-6699 / E-mail: sady@unochapeco.edu.br

Submissão: 07/08/2020. Revisão: 05/11/2020. Aceite: 26/11/2020. Publicação: 29/12/2020.

DOI: http://dx.doi.org/10.22277/rgo.v14i1.5715

\section{RESUMO}

A expansão da pandemia COVID-19 desestruturou o cenário econômico mundial, se alastrou rapidamente causando mortes e modificando as relações sociais. $O$ estudo tem por objetivo analisar os fatores sociais, econômicos, demográficos e culturais que influenciam na propagação da COVID-19 e mortalidade das pessoas em âmbito internacional. A análise dos dados de 110 países ocorreu pelo uso das técnicas de estatística descritiva e regressão linear múltipla. Os resultados apontam que as variáveis determinantes da COVID-19 (casos confirmados, mortes, recuperados, índice de contaminação e índice de mortalidade) podem ser explicadas, isoladamente, tanto por fatores sociais, econômicos e demográficos, quanto pelo comportamento característico da cultura de cada nação. Constatou-se números de casos maiores em países mais corruptos, com maior índice de desemprego, maior tamanho demográfico, maior PIB e com culturas predominantemente individualistas e masculinas. $\mathrm{O}$ número de mortes foi mais constatado em países mais corruptos, populosos, com PIB mais elevado e com cultura individualista. Por sua vez, o número de recuperados foi mais consistente em países com poder econômico mais elevado e cultura de aversão a incerteza.

Palavras-chave: COVID-19. Coronavírus. Fatores Culturais. Fatores sociais. 


\section{ABSTRACT}

The expansion of the COVID-19 pandemic has unstructured the world economic scene, spread rapidly causing deaths and changing social relations. The study aims to analyze the social, economic, demographic, and cultural factors that influence the spread of COVID-19 and mortality of people internationally. Data analysis from 110 countries was performed using descriptive statistics and multiple linear regression techniques. The results indicate that the determinant variables of COVID-19 (confirmed cases, deaths, recoveries, contamination index and mortality rate) can be explained, separately, both by social, economic, and demographic factors, as well as for the characteristic behavior of each national culture. There were larger numbers of cases in more corrupt countries, with higher unemployment rate, larger population size, higher GDP, and predominantly individualistic and male cultures. The number of deaths was more observed in more corrupt, populous countries with higher GDP and individualistic culture. In turn, the number of recoveries was more consistent in countries with higher economic power and culture of aversion to uncertainty.

Keywords: COVID-19. Coronavírus. Cultural factors. Social factors.

\section{INTRODUÇÃO}

Inúmeras crises já assolaram o mundo, desde a Peste Negra (século XIV), a Gripe de 1918, os períodos pós-guerra e os colapsos financeiros. Esses episódios foram responsáveis por ocasionar a morte de inúmeras pessoas e por gerar impactos sociais, tendo consequências na política, na economia e na vida das comunidades (GAMA NETO, 2020; RITA; FERREIRA JUNIOR, 2020; AVENI, 2020; MURSHED, 2020).

Porém, apesar de se distinguirem por serem pandemias virais ou crises financeiras, possuem como característica semelhante a interferência de ações e a necessidade de políticas públicas, pois impactam na renda, na geração de empregos, nas condições de moradia, alimentação e saúde (LEMOS; ALMEIDA FILHO; FIRMO, 2020).

A pandemia mais recente, a COVID-19, é causada pelo vírus SARS-CoV-2 e surgiu no final do ano de 2019 na cidade de Wuhan, província de Hubei na China (GAMA NETO, 2020; KRAEMER et al., 2020; OMS, 2020; OPA, 2020). Em decorrência do crescimento no número de casos de forma exponencial e o fato de ter se alastrado pelo mundo rapidamente com um alto número de infectados, foi declarada pela Organização Mundial de Saúde (OMS) como pandemia mundial (FERREIRA JUNIOR; RITA, 2020; OMS, 2020).

A mortalidade súbita de milhares de pessoas exigiu investimentos para as delongadas internações e no processo de recuperação de pacientes, além de medidas de isolamento social e restrições comerciais entre as nações, que se refletiram no mercado econômico e financeiro (FERREIRA JUNIOR; RITA, 2020). Ainda não se sabe quando, nem como irá terminar esta nova pandemia, mas em poucos meses já se instalou um cenário caótico, que desestruturou as perspectivas econômicas mundiais para o ano de 2020 e possivelmente se refletirá nos próximos anos.

A pandemia global da COVID-19 não causa apenas infecções e mortes, mas passa a evidenciar as limitações dos sistemas de saúde (AVENI, 2020), além de ocasionar profundos impactos socioeconômicos em uma escala não vista desde a Grande Depressão dos anos de 1930 e da Crise da Bolha Imobiliária de 2008 (MARANHÃO; SENHORAS, 2020; GAMA NETO, 2020). As repercussões em escala mundial deixaram evidências do despreparo e a falta de 
coordenação conjunta dos países para minimizar as consequências da doença (GAMA NETO, 2020).

Frente ao cenário de contaminação e com o intuito de minimizar o colapso dos sistemas de saúde e a propagação da doença, os governos passaram a adotar inúmeras medidas, desde o maior distanciamento social, isolamento, quarentena e fechamento de fronteiras (GAMA NETO, 2020; LEMOS; ALMEIDA FILHO; FIRMO, 2020; CEPAL, 2020). Medidas estas, que refletiram em impactos em todas as nações e suas economias (GAMA NETO, 2020; CEPAL, 2020). E apesar de países como a China, Japão e Taiwan, terem se demonstrados mais preparados para tal desastre e terem conseguido inicialmente controlar de forma rápida a disseminação da doença, também sofreram impactos econômicos e sociais (GAMA NETO, 2020; HUYNH, 2020).

De maneira geral, as medidas adotadas para a contenção do vírus, foi semelhante entre todas as nações, no entanto, os resultados de tais medidas mostraram-se distintos em cenários em que a população possui acesso aos serviços de saúde de forma gratuita e proteção à renda, com outros ambientes em que as pessoas acabam tendo que optar em trabalhar para manter seu sustento (LEMOS; ALMEIDA FILHO; FIRMO, 2020). Essa distinção ocorre devido às diferenças sociais, econômicas, demográficas e culturais que existem entre as nações do mundo.

No ambiente das organizações, os impactos dessas medidas refletiram níveis de retornos mais baixos aos acionistas, menores investimentos em indústrias e redução da eficiência organizacional, logo, os impactos serão significativos na esfera privada, sobretudo na geração de riqueza, abrindo espaço para a inadimplência e a impossibilidade de cumprimento de obrigações de curto prazo (MARANHÃO; SENHORAS, 2020).

Assim, na perspectiva de Nicola et al. (2020), o COVID-19 afeta não apenas as comunidades, mas também impacta nas empresas, nos mercados financeiros e nas economias globais. Por exemplo, na China, as restrições quanto aos bloqueios acabaram por reduzir significativamente a produção de bens e serviços, porém, o fato de ter realizado o isolamento anterior aos demais países, está suscetível a recuperação do mercado em menor tempo, aumentando seu poder de negociação com os países ocidentais. Nesse sentido, a escala dos impactos ocasionados pelo vírus, estão atrelados aos aspectos de saúde e decorrem basicamente da rapidez como os governos lidam com as implicações financeiras que se instauram a partir das restrições sociais e econômicas (BHUSAL, 2020; SOUZA, 2020).

Frente a esse cenário tem-se a seguinte problemática de estudo: quais os fatores sociais, econômicos, culturais e demográficos influenciam na propagação dos casos de COVID19 e na mortalidade de pessoas em âmbito internacional? O objetivo do estudo consiste em analisar os fatores sociais, econômicos, culturais e demográficos que influenciam na propagação dos casos de COVID-19 e mortalidade das pessoas em âmbito internacional.

Para responder ao problema e alcançar o objetivo proposto, utilizou-se a estratégia metodológica de pesquisa quantitativa, coletando-se os casos registrados de COVID-19 de 110 países, conforme divulgação do World Bank (2020). Os aspectos culturais foram coletados da base Hofstede, e os fatores sociais e econômicos coletados de distintas fontes internacionais. Como análise complementar, considerou-se a amostra dos 25 países que mais relataram casos confirmados do coronavírus. A técnica de análise utilizada foi a regressão linear múltipla.

O estudo justifica-se em decorrência do cenário em que todas as nações do mundo foram obrigadas a fazer escolhas para conter o avanço da doença e minimizar os impactos sociais, econômicos e financeiros. Porém, as medidas econômicas adotadas diferem entre os países, como consequência, o avanço do vírus e o número de mortes não seguem o mesmo 
padrão entre as nações. Dessa forma, verifica-se a existência de fatores ou variáveis distintas (sociais, econômicas, demográficas e culturais) de cada nação, que podem justificar os níveis diferentes de propagação, contenção e mortalidade ocasionada pela COVID-19.

É relevante estudar a influência das culturas sobre a pandemia, pois algumas culturas são mais obedientes que outras, levando as pessoas a seguirem melhor as medidas de isolamento mais rigorosas, recomendadas universalmente para conter o contágio (FREY; CHEN, PRESIDENTE, 2020). Nesse sentido, o estudo contribui ao apresentar evidências de que a cultura de cada nação está intrinsecamente relacionada com o sucesso ou o fracasso das medidas impostas internacionalmente na tentativa de conter o avanço do vírus. $O$ sucesso na contenção dos casos dependerá da combinação de ações governamentais que levem em conta as deficiências econômicas e sociais, os fatores demográficos de grandes países que impedem o controle total de suas fronteiras e os fatores culturais de sua população, que pode representar uma barreira importante para o enfrentamento da pandemia.

Espera-se que a partir do conhecimento dos fatores influenciadores do aumento do contágio e mortalidade, seja possível contribuir com reflexões acerca de possíveis soluções e perspectivas futuras para o enfrentamento da COVID-19 e novas pandemias, reduzindo o impacto negativo sobre os meios de subsistência, dos negócios e das economias mundiais (LAING, 2020; FERREIRA JUNIOR; RITA, 2020; GAMA NETO, 2020). Assim como, almeja-se cooperar com a literatura sobre a temática, a qual apresenta lacunas de estudos cross-country com a inserção de variáveis sociais, econômicas, demográficas e culturais.

Convém ressaltar que este estudo não pretende realizar posicionamentos políticos e inferir ou analisar o conjunto de medidas governamentais adotadas por cada país, isso pois, muitas destas, ainda estão em formulação, aprovação e regulamentação pelas autoridades competentes e são adotadas de formas distintas por cada país. O intuito é verificar as relações existentes entre as variáveis socioeconômicas, demográficas e culturais e como estas influenciam nos números ligados à doença.

\section{CARACTERÍSTICAS SOCIOECONÔMICAS, CULTURAIS E DEMOGRÁFICOS DOS PAÍSES}

Apesar das capacidades produtivas e financeiras não terem sofrido colapsos econômicos semelhantes aos períodos de pós-guerras ou grandes recessões, o tempo de permanência dos bloqueios pode ocasionar na redução da demanda de produtos e na redução de preços. Consequentemente, em uma perspectiva de médio prazo, os investimentos organizacionais podem se tornar irrecuperáveis, devido a redução dos lucros ou as perdas em determinados setores. Isso poderia ser traduzido em menores retornos aos acionistas e baixos investimentos nas indústrias, impactando a eficiência produtiva. Consequentemente acaba por influenciar na redução de valores destinados a projetos comunitários, sociais e ambientais, além de acarretar pressões nos salários das famílias, afetando o meio de subsistência e gerando menores receitas fiscais aos governos (LAING, 2020; CEPAL, 2020).

Outro impacto decorrente da pandemia está atrelado a ruptura nas cadeias de suprimentos (SENHORAS, 2020; GAMA NETO, 2020; MURSHED, 2020), em decorrência da condição global e integrada da economia, além do impacto negativo nos mercados de capitais, com a queda dos rendimentos e níveis críticos de liquidez, até a adoção por parte dos governos de pacotes de medidas de auxílios econômicos, frente a perda da renda afetada pelo coronavírus (NICOLA et al., 2020). O impacto da COVID-19 pode ser vislumbrado nas bolsas dos EUA, Alemanha, nos Mercados asiáticos e na Europa (NICOLA et al., 2020). Visualiza-se 
nesse cenário, a ausência de coordenação de políticas internacionais e a falta de liderança econômica em países como, por exemplo, os Estados Unidos (MURSHED, 2020).

Todos os países sofreram ou sofrerão os impactos da COVID-19. Variáveis como produto interno bruto, situação fiscal e estruturas econômicas de cada país podem ser determinantes para a proporção do impacto causado pelo vírus (GAMA NETO, 2020). Assim, países mais desenvolvidos, com melhores condições de políticas de saúde e maior renda per capita, podem ter maiores chances de superar a crise, em relação aos países subdesenvolvidos, em que o aumento dos níveis de desemprego eleva o aumento da pobreza, conforme dados da Comissão Econômica para a América Latina e o Caribe (CEPAL, 2020).

Porém, para Furtado (2009) os países subdesenvolvidos não estão fadados à dependência e ao atraso, pois o subdesenvolvimento é uma formação histórica específica e passível de superação, uma vez que faz parte de uma etapa histórica do desenvolvimento de um país. Para evitar o aumento do desemprego países subdesenvolvidos como o Brasil, Argentina, México, Chile, Peru e Colômbia, passaram a realizar aportes de recursos para a população, flexibilizaram a redução de jornada de trabalho e criaram fundos especiais para o combate à doença, além de estímulos fiscais para evitar o colapso econômico (GAMA NETO, 2020).

No entanto, as medidas governamentais para evitar um impacto maior pela COVID-19 na economia, acaba aumentando a dívida pública (BRESSER-PEREIRA, 2020). Para vários autores existe uma correlação negativa entre dívida pública e crescimento econômico, que se torna particularmente forte quando a dívida pública se aproxima de 100\% do PIB (REINHART; ROGOFF, 2010; KUMAR; WOO, 2010; CECCHETTI; MOHANTY; ZAMPOLLI, 2011), e isso seria catastrófico para países que já enfrentavam antes da pandemia complexos problemas econômicos.

Por sua vez, os Estados Unidos, tornaram-se rapidamente o país com maior número de infectados pelo vírus mundialmente, mesmo sendo classificado como país desenvolvido. $\mathrm{E}$, apesar de adotar estratégias semelhantes de outras nações atreladas ao isolamento e paralisação das atividades não essenciais, convive com o aumento do desemprego, o qual passou de 4,4\% para 11\% em pouco mais de um mês (HUYNH, 2020; GAMA NETO, 2020).

De acordo com avaliação da Organização Internacional do Trabalho (OIT), o impacto direto da COVID-19 provocará aumento do desemprego em âmbito global, atingindo 25 milhões de pessoas, com estimativa de atingir 35 milhões a mais de pessoas trabalhando na pobreza após a pandemia, em comparação com a estimativa original mundial para 2020. Conforme as Nações Unidas Brasil (2020), se levado em conta os diferentes cenários para o impacto da COVID-19 no crescimento do PIB global, as estimativas da OIT indicam um aumento no desemprego global entre 5,3 milhões (cenário de baixo impacto) e 24,7 milhões (cenário de alto impacto), a partir de um nível base de 188 milhões em 2019. Essa perda de rendimentos se traduzirá no menor consumo de bens e serviços, e levando muitos trabalhadores a situações de pobreza (CEPAL, 2020).

Nesse ambiente, é preciso entender os fatores e às medidas de contenção da doença, pois o isolamento social reduz a circulação de dinheiro e respectivamente as receitas tributárias, as quais são fundamentais para as finanças e manutenção da saúde pública (LEMOS; ALMEIDA FILHO; FIRMO, 2020; FERREIRA JUNIOR; RITA, 2020). Por sua vez, também acarreta a diminuição das jornadas de trabalhos ou salários, e até mesmo no desemprego. Consequentemente, reduz a geração de renda das famílias, afetando diretamente as receitas das organizações e toda a cadeia de valor envolvida, uma vez que se tem a diminuição da demanda agregada de bens e serviços (LEMOS; ALMEIDA FILHO; FIRMO, 2020; FERREIRA 
JUNIOR; RITA, 2020; CEPAL, 2020). Em contrapartida, a formação bruta de capital das empresas é afetada de forma adversa, impactando na falta de investimentos (CEPAL, 2020).

O problema é que quando os países mais ricos são os mais afetados, pode haver um impacto maior ainda quanto aos níveis de desigualdade. Os registros históricos demonstram que o capitalismo tende a criar um círculo vicioso de desigualdade, pois, no longo prazo, a taxa de retorno sobre os ativos é maior que o ritmo do crescimento econômico, o que se traduz numa concentração cada vez maior da riqueza (PIKETTY, 2014).

Nessa perspectiva, a crise terá impacto negativo na saúde e na educação, bem como no emprego e na pobreza. Haverá fortes impactos no setor de saúde devido à escassez de mão de obra qualificada e suprimentos médicos, bem como aumentos de custos. A maioria dos países possui sistema de saúde fracos que não garantem o acesso universal a saúde, necessário ao enfrentamento da doença, sendo em inúmeras vezes desigual para determinados grupos populacionais (CEPAL, 2020). Associado a isso, muitos sistemas de saúde ainda se encontram centralizados geograficamente, ou seja, com serviços médicos especializados em poucos centros urbanos e com instalações e equipamentos insuficientes para o nível de demanda (CEPAL, 2020; FREITAS; SILVA; CIDADE, 2020).

Quanto à educação, a interrupção das atividades nos centros de ensino terá efeitos negativos no aprendizado, principalmente para os indivíduos mais vulneráveis. Muitas crianças dependem da segurança alimentar e cuidados fornecidos pelas escolas, o que permite a família trabalhar e obter renda para o sustento. Embora, muitas instituições adotaram estratégias para o ensino remoto, é visível que nem todas possuem infraestrutura e pessoal capacitado para atuar nessa nova metodologia. Da mesma forma, existe disparidade quanto ao acesso de tecnologias e redes de internet de qualidade para a população (CEPAL, 2020).

Lancker e Parolin (2020) corroboram ao apontar que o fechamento das escolas afeta ainda mais as crianças mais pobres em dois pontos cruciais, primeiro, em relação a alimentação, pois muitas delas tem a escola como maior fonte de nutrição, e em segundo quando a pesquisa sugere que os fatores escolares são a principal fonte de desigualdade social.

Ademais, fatores como a corrupção tende a aumentar no setor da saúde com a injeção de grandes quantias de dinheiro para aliviar o estado de emergência e a crise por leitos, atendimento e suprimentos médicos (KRASTEV; PETROV; MARINOV, 2020). Para tanto, faz-se necessário meios para garantir a transparência no poder público, como principal forma de prevenção a corrupção, visto que diante do cenário as regras de contratação dos bens e serviços passam a ser simplificados (GALLEGO; PREM; VARGAS, 2020; KRASTEV; PETROV; MARINOV, 2020).

Por conseguinte, estudos demonstram que a densidade urbana também está relacionada positivamente com o número de casos atuais da COVID-19. A má qualidade de habitação de inúmeros indivíduos impacta e pode desencadear uma problemas de saúde, como asma e demais doenças respiratórias, consequentemente contribuindo no agrave da situação (HU et al., 2020)

Outros aspectos, como as características culturais de cada nação também podem interferir na forma de avanço e contaminação da COVID-19. Na avaliação de Anderson et al. (2020), a única coisa que os humanos podem fazer para interromper o surto de COVID-19 é mudar seus comportamentos, incorporando a adoção das recomendações de distanciamento social e higienização. Todavia, isso mostra-se um desafio frente às diferenças culturais 
Dyeniffer Packer Eigenstuhler, Francieli Pacassa, Silvana Dalmutt Kruger e Sady Mazzioni

existentes nos diferentes países e o fato dos indivíduos terem percepções distintas a respeito da doença (HUYNH, 2020).

O Quadro 1 apresenta as dimensões culturais que podem explicar o comportamento das sociedades frente aos mais diversos aspectos.

Quadro 1 - Variáveis de cultura

\begin{tabular}{|c|c|}
\hline Cultura Nacional & Métrica e Conceito \\
\hline Distância do Poder & $\begin{array}{l}\text { Escala de } 0 \text { a 100, em que mais próxima de } 100 \text { maior a distância do poder (maior } \\
\text { desigualdade, hierarquização, superiores inacessíveis, privilégios para quem tem mais } \\
\text { poder, as crianças aprendem a obedecer) e, mais próxima de } 0 \text { menor a distância do } \\
\text { poder (desigualdade minimizada, hierarquia apenas por conveniência, superiores } \\
\text { acessíveis, todos têm os mesmos direitos, as crianças são tratadas com igualdade). }\end{array}$ \\
\hline $\begin{array}{c}\text { Individualismo/ } \\
\text { Coletivismo }\end{array}$ & $\begin{array}{l}\text { Escala de } 0 \text { a 100, em que mais próxima de } 100 \text { maior o individualismo do país (foco no } \\
\text { "eu", ênfase nas escolhas pessoais, cumprir as próprias obrigações, livre expressão direta, } \\
\text { pouco importância para a comunicação) e, mais próxima de } 0 \text { maior o coletivismo (foco } \\
\text { no coletivo, os relacionamentos importam mais que as tarefas, cumprir as obrigações } \\
\text { impostas pelo grupo, manter a harmonia, a comunicação é importante). }\end{array}$ \\
\hline Aversão à Inc & $\begin{array}{l}\text { Escala de } 0 \text { a } 100 \text {, em que mais próxima de } 100 \text { maior aversão à incerteza (alto estresse } \\
\text { em situações de incerteza, a incerteza é uma ameaça que deve ser combatida, } \\
\text { necessidade de consenso, evita-se o fracasso, grande necessidade de regras e leis) e, mais } \\
\text { próxima de } 0 \text { menor aversão à incerteza (baixo estresse em situações de incerteza, a } \\
\text { incerteza é aceita como parte da vida, as coisas são aceitas como são, as diferenças de } \\
\text { opinião são aceitáveis, se sentem bem correndo riscos, pouca necessidade de regras e } \\
\text { leis). }\end{array}$ \\
\hline $\begin{array}{l}\text { Masculinidade/ } \\
\text { Feminilidade }\end{array}$ & $\begin{array}{l}\text { Escala de } 0 \text { a } 100 \text {, em que mais próxima de } 100 \text { maior a masculinidade do país (ambição, } \\
\text { viver para trabalhar, admiração pelo que é grande e pelo sucesso, os mais fortes vencem } \\
\text { os conflitos) e, mais próxima de } 0 \text { maior a feminilidade (foco na qualidade de vida, } \\
\text { trabalhar para viver, admiração pelas coisas pequenas e agradáveis, compaixão pelos } \\
\text { menos afortunados, os conflitos são resolvidos através do compromisso e da } \\
\text { negociação). }\end{array}$ \\
\hline $\begin{array}{l}\text { Orientação Longo } \\
\text { Prazo/Curto Prazo }\end{array}$ & $\begin{array}{l}\text { Escala de } 0 \text { a } 100 \text {, em que mais próxima de } 100 \text { maior a orientação em longo prazo (os } \\
\text { resultados são mais lentos, é importante economizar e ser cuidadoso com os recursos, } \\
\text { disposição para adiar os desejos pessoais por uma boa causa) e, mais próxima de } 0 \text { maior } \\
\text { a orientação em curto prazo (os resultados devem ser imediatos, há uma pressão social } \\
\text { para gastar mais, os lucros imediatos são mais importantes que as relações). }\end{array}$ \\
\hline $\begin{array}{l}\text { Complacência/ } \\
\text { Repressão }\end{array}$ & $\begin{array}{l}\text { Escala de } 0 \text { a } 100 \text {, em que mais próxima de } 100 \text { maior a complacência (liberdade, as } \\
\text { recompensas materiais não são importantes, centro no presente, maior valor a utilidade } \\
\text { e não ao status, pessoas otimistas, positivas, extrovertidas, simpáticas, valorizam o lazer } \\
\text { e os amigos) e, mais próxima de } 0 \text { maior a repressão (comportamentos suprimidos e } \\
\text { regulados, espera-se recompensa material, sentimento de injustiça, os bens e objetos são } \\
\text { importantes para o status, as pessoas são mais pessimistas, reservadas e o lazer e as } \\
\text { amizades não são tão importantes) }\end{array}$ \\
\hline
\end{tabular}

Fonte: adaptado de Hofstede (2011).

As diferenças culturais podem conduzir a relação estabelecida entre médico-paciente, a estrutura do sistema de saúde e o treinamento dos profissionais de saúde (MORROW et al., 2013). Desse modo, as dimensões culturais apresentadas no Quadro 1 podem explicar os mecanismos de controle (adotados ou não) para uma doença infecciosa como a COVID-19. A cultura é um fenômeno coletivo de programação mental que distingue os indivíduos pertencentes a determinados grupos e sociedades (HOFSTEDE, 2011). As dimensões de cultura de Hofstede (2011), demonstram fatores preditivos para observar os diferentes 
comportamentos, que podem ser vinculados para explicar a condução das medidas de combate e prevenção da propagação do coronavírus.

Por meio das dimensões de cultura, pode-se inferir que haverá países com uma cultura mais restritiva no enfrentamento de desastres, surtos e a densidade populacional e outros que terão uma cultura mais frouxa em relação às medidas a serem adotadas, priorizando a individualidade (HUYNH, 2020). Gorodnichenko e Roland (2015) argumentam que países coletivistas são mais capazes de resolver problemas de ação coletiva, como montar uma resposta coordenada a uma pandemia, já os países com cultura individualista teriam muito mais dificuldade para isso.

Assim, a heterogeneidade das determinantes culturais entre as diferentes nações pode desempenhar papel relevante no controle da COVID-19, principalmente quando atrelado ao comportamento dos indivíduos em relação ao distanciamento social, o qual temse evidenciado um dos melhores conselhos pelas autoridades para conter a propagação do vírus (HUYNH, 2020). Não obstante, mostra-se relevante a realização de planejamento e desenvolvimento de planos socioeconômicos pelos governos, instituições financeiras e organizações, para possibilitar a manutenção de modelos de negócios sustentáveis e incentivar o empreendedorismo (NICOLA et al., 2020), para minimizar os impactos econômicos negativos.

\section{PROCEDIMENTOS METODOLÓGICOS}

Metodologicamente a pesquisa se caracteriza como descritiva, realizada a partir de análise documental, com cunho quantitativo. A população da pesquisa corresponde a 193 nações que possuíam casos registrados de COVID-19 na data da coleta (05/06/2020), conforme dados do World Bank (2020). Para a definição da amostra investigada foram excluídos os países que não possuíam dados referentes ao número de casos, mortes e recuperados da COVID-19 e aqueles que não possuíam dados na base Hofstede, que mensura a cultura dos países. Com os procedimentos adotados, a amostra final do estudo ficou composta por 110 nações.

A estruturação da análise foi elaborada levando em consideração dois grupos de países: o grupo com a amostra total (110 países) e o grupo dos 25 países que mais relataram casos confirmados do coronavírus. Justifica-se a escolha da análise isolada dos 25 países que mais relataram casos, considerando que juntos somam $90,06 \%$ de todos os casos confirmados no mundo e $95,64 \%$ de todas as mortes ocorridas pela COVID-19, conforme demonstra a Tabela 1.

Tabela 1 - Relevância dos 20 países mais contaminados

\begin{tabular}{l|c|c}
\hline Casos & Confirmados & Mortes \\
\hline Total Global & 6.416 .828 & 382.867 \\
20 países mais contaminados* & 5.779 .108 & 366.202 \\
Representatividade \% dos 20 países mais contaminados & $90,06 \%$ & $95,64 \%$ \\
\hline
\end{tabular}

Amostra: Estados Unidos, Brasil, Rússia, Reino Unido, Espanha, Itália, Índia, Alemanha, Peru, Turquia, Irã, França, Chile, México, Arábia Saudita, Canadá, Paquistão, China, Catar e Bangladesh.

Fonte: dados da pesquisa (2020).

Para a análise dos dados utilizou-se da regressão linear múltipla, a partir das variáveis dependentes e independentes elencadas na Tabela 2. 
Dyeniffer Packer Eigenstuhler, Francieli Pacassa, Silvana Dalmutt Kruger e Sady Mazzioni

Tabela 2 - Construto das Variáveis Dependentes e Independentes

\begin{tabular}{|c|c|c|c|c|}
\hline \multicolumn{5}{|c|}{ Variáveis dependentes } \\
\hline & \multicolumn{2}{|r|}{ Descrição } & \multicolumn{2}{|r|}{ Fonte } \\
\hline \multirow{5}{*}{$\begin{array}{c}\text { Variáveis } \\
\text { dependentes }\end{array}$} & \multicolumn{2}{|c|}{ Casos Confirmados } & \multicolumn{2}{|r|}{ World Bank } \\
\hline & \multicolumn{2}{|c|}{ Casos recuperados } & \multicolumn{2}{|r|}{ World Bank } \\
\hline & \multicolumn{2}{|r|}{ Mortes } & \multirow{2}{*}{\multicolumn{2}{|c|}{ World Bank }} \\
\hline & \multicolumn{2}{|c|}{ Índice de Contaminação } & & \\
\hline & \multicolumn{2}{|c|}{ Índice de Mortalidade } & \multicolumn{2}{|r|}{ Mortes/População } \\
\hline \multicolumn{5}{|c|}{ Variáveis independentes } \\
\hline & Sigla & $\mathrm{De}$ & & Fonte \\
\hline \multirow{3}{*}{$\begin{array}{l}\text { Variáveis } \\
\text { Sociais }\end{array}$} & TXALF & \multicolumn{2}{|c|}{ Taxa de Alfabetização (\%) } & The World FactBook \\
\hline & DESEMP & \multicolumn{2}{|c|}{ Desemprego } & Trading Economics \\
\hline & PERCCOR & \multicolumn{2}{|c|}{ Percepção da Corrupção } & $\begin{array}{l}\text { Transparência Internacional } \\
\text { Brasil }\end{array}$ \\
\hline \multirow{3}{*}{$\begin{array}{c}\text { Variáveis } \\
\text { Demográficas }\end{array}$} & TAMKM & \multicolumn{2}{|c|}{ Tamanho territorial em $\mathrm{Km}^{2}$} & The World FactBook \\
\hline & DENSPOP & \multicolumn{2}{|c|}{ Densidade Populacional } & População/Tamanho $\mathrm{Km}^{2}$ \\
\hline & POPUL & \multicolumn{2}{|c|}{ População } & The World FactBook \\
\hline \multirow{4}{*}{$\begin{array}{l}\text { Variáveis } \\
\text { Econômicas }\end{array}$} & IDH & \multicolumn{2}{|c|}{ Índice de Desenvolvimento Econômico } & PNUD \\
\hline & PIB & \multicolumn{2}{|c|}{ PIB } & $\begin{array}{l}\text { International Monetary } \\
\text { Fund }\end{array}$ \\
\hline & PIBPC & \multicolumn{2}{|c|}{ PIB per capita } & PIB/População \\
\hline & DIVPUB & \multicolumn{2}{|c|}{ Dívida Pública } & Trading Economics \\
\hline \multirow{6}{*}{$\begin{array}{l}\text { Variáveis de } \\
\text { Cultura }\end{array}$} & DISTPOD & \multicolumn{2}{|c|}{ Distância do Poder } & \multirow{6}{*}{ Hofstede Insights } \\
\hline & INDIVID & Individualism & & \\
\hline & MASCUL & Masculinidad & & \\
\hline & AVINC & Aversão & & \\
\hline & ORILPRAZO & \multicolumn{2}{|c|}{ Orientação à Longo Prazo ou Curto Prazo } & \\
\hline & COMPLAC & \multicolumn{2}{|c|}{ Complacência ou Repressão } & \\
\hline
\end{tabular}

Fonte: dados da pesquisa (2020).

A coleta dos dados coletados (Tabela 2) referentes às variáveis independentes de cultura, as quais podem influenciar nos níveis de contaminação e mortalidade, ocorreu de forma manual, nas fontes elencadas. A análise de dados ocorreu pelo uso das técnicas de estatística univariada, bivariada e multivariada, por meio do software STATA. Para validar os resultados das regressões foram observados os pressupostos de normalidade; multicolinearidade, por meio do Fator de Inflação da Variância (VIF); de homocedasticidade e ausência de autocorrelação serial, por meio do teste de Durbin-Watson. Os dados são apresentados por meio da análise das variáveis, combinadas ainda com os gráficos de evolução dos casos confirmados por país, para que assim houvesse uma melhor compreensão visual das discrepâncias entre as nações analisadas.

\section{DISCUSSÃO E ANÁLISE DE DADOS}

Esta seção contém a descrição e análise dos dados. Primeiramente apresentam-se informações à despeito dos países mais afetados pelas variáveis dependentes no período de análise. Em seguida apresenta-se os resultados das regressões, que possibilitaram verificar o poder preditivo das variáveis sociais, demográficas, econômicas e culturais na influência, propagação e mortalidade da COVID-19

A Tabela 3 mostra o ranking dos países quanto ao número de casos confirmados, recuperados, mortes, recuperabilidade, contaminação e mortalidade, da amostra formada por 110 nações, onde é possível constatar que os Estados Unidos é o país com o maior número 
de casos confirmados e o maior número de mortes. Porém, quando analisado os índices de mortalidade, recuperabilidade e contaminação, não ocupam mais a primeira posição.

Tabela 3 - Ranking dos Países por variável dependente

\begin{tabular}{|c|c|c|c|c|c|c|}
\hline Ranking & Confirmados & Recuperados & Mortes & $\begin{array}{c}\text { Índice } \\
\text { Recuperabilidade }\end{array}$ & $\begin{array}{c}\text { Índice } \\
\text { Contaminação }\end{array}$ & $\begin{array}{c}\text { Índice } \\
\text { Mortalidade }\end{array}$ \\
\hline 10 & $\begin{array}{l}\text { Estados } \\
\text { Unidos }\end{array}$ & $\begin{array}{l}\text { Estados } \\
\text { Unidos }\end{array}$ & $\begin{array}{l}\text { Estados } \\
\text { Unidos }\end{array}$ & Islândia & Qatar & França \\
\hline 20 & Brasil & Brasil & $\begin{array}{l}\text { Reino } \\
\text { Unido }\end{array}$ & Nova Zelândia & Kuwait & Bélgica \\
\hline 3응 & Rússia & Rússia & Brasil & Montenegro & Singapura & Itália \\
\hline 40 & Reino Unido & Alemanha & Itália & Taiwan & Luxemburgo & Reino Unido \\
\hline 5운 & Espanha & Itália & França & Luxemburgo & Chile & Hungria \\
\hline
\end{tabular}

Fonte: dados da pesquisa (2020).

Os maiores índices de recuperabilidade (recuperados/número de confirmados) estão em países menores e com IDH mais elevado (Islândia e Nova Zelândia). Por outro lado, os maiores índices de contaminação (número de casos/população) estão no Catar e Kuwait. Estes correspondem a países pequenos e que possuem, respectivamente, $67,7 \%$ e $74,6 \%$ de sua população declarada como muçulmana (CIA WORLD FACTBOOK, 2020), denotando possuir culturas que se diferenciam das demais nações em análise. Já os maiores índices de mortalidade estão na França, Bélgica e Itália, que são países vizinhos no continente europeu e que possuem uma população mais idosa, além de uma cultura predominantemente individualista, corroborando os indicativos de Gorodnichenko e Roland (2015). Na Tabela 4 apresenta-se os dados das regressões com as variáveis independentes relacionadas ao vírus.

Tabela 4 - Resultados da regressão da amostra total de nações

\begin{tabular}{|c|c|c|c|c|c|}
\hline \multirow{3}{*}{$\begin{array}{c}\text { Variáveis } \\
\text { Independentes }\end{array}$} & \multicolumn{5}{|c|}{ Variável dependente } \\
\hline & Confirmados & Mortes & Recuperados & $\begin{array}{c}\text { Índice de } \\
\text { Contaminação }\end{array}$ & $\begin{array}{c}\text { Índice de } \\
\text { Mortalidade }\end{array}$ \\
\hline & Coeficiente & Coeficiente & Coeficiente & Coeficiente & Coeficiente \\
\hline TXALF & $-87.680,450$ & $-7.923,841$ & $-36.236,840$ & $0,004 * * *$ & 0,011 \\
\hline PIBPC & 0,151 & $-0,015$ & 0,338 & $1,030 *$ & 5,660 \\
\hline POPUL & $-0,000 *$ & $-0,000 *$ & $-0,000 *$ & 4,260 & 2,450 \\
\hline IDH & 94,676 & 10,445 & 81,911 & $-2,240$ & 0,000 \\
\hline PIB & $0,063 *$ & $0,004 *$ & $0,012 *$ & 1,011 & 1,020 \\
\hline TAMKM & $0,020 *$ & 0,000 & $0,009 *$ & 1,401 & $-9,990$ \\
\hline DENSPOP & $-549.038,600 * *$ & - & $-202.651,200 *$ & $-0,007 * * *$ & $-0,107 * * *$ \\
\hline DESEMP & $2.839,819$ & 129,082 & $1.059,022$ & 0,000 & 0,000 \\
\hline DIVPUB & $-121,356$ & 19,678 & 15,300 & 9,370 & $0,000 * *$ \\
\hline PERCCORR & $-1.227,705$ & $-105,131$ & $-778,155 * * *$ & 0,000 & 0,000 \\
\hline DISTPODER & 386,228 & 50,695 & $-50,245$ & $0,001 *$ & 0,000 \\
\hline INDIVID & $2.290,175^{* *}$ & 239,310* & $599,365 * *$ & $-9,850$ & $0,001 *$ \\
\hline MASCUL & $-827,786$ & $-47,654$ & $-136,379$ & 0,000 & 0,000 \\
\hline AVINC & 453,322 & 19,486 & 327,909 & 0,000 & $0,000 * * *$ \\
\hline ORILPRAZO & $-943,124 * * *$ & $-49,885$ & $-131,291$ & $-0,000 *$ & 0,000 \\
\hline INDULG & 88,754 & 30,084 & $-72,728$ & $-0,000 * * *$ & 0,000 \\
\hline Constante & $2.206,445$ & $-6.310,197$ & $-22.232,87$ & $-0,004 * * *$ & $-0,018$ \\
\hline Estatística F & 12,37 & 11,46 & 10,28 & 4,86 & 3,76 \\
\hline$R^{2}$ & 0,6804 & 0,6634 & 0,6389 & 0,4555 & 0,3930 \\
\hline VIF & 2,58 & 2,58 & 2,58 & 2,58 & 2,58 \\
\hline DW & 1,93 & 1,72 & 1,95 & 2,00 & 2,25 \\
\hline $\mathrm{N}$ & 110 & 110 & 110 & 110 & 110 \\
\hline
\end{tabular}

Legenda: ${ }^{*}$ significativo a $1 \% ;{ }^{* *}$ significativo a $5 \% ; * * *$ Significativo a $10 \%$. 
Dyeniffer Packer Eigenstuhler, Francieli Pacassa, Silvana Dalmutt Kruger e Sady Mazzioni

Amostra: África do Sul, Albânia, Alemanha, Angola, Arábia Saudita, Argélia, Argentina, Armênia, Austrália, Azerbaijão, Bangladesh, Bélgica, Bielorússia, Bolívia, Brasil, Bulgária, Canadá, Cazaquistão, Chile, China, Colômbia, Coreia do Sul, Costa Rica, Croácia, Dinamarca, Egito, El Salvador, Emirados Árabes, Equador, Eslováquia, Eslovênia, Espanha, Estados Unidos, Estônia, Etiópia, Filipinas, Finlândia, França, Gana, Geórgia, Grécia, Guatemala, Holanda, Honduras, Hong Kong, Hungria, Índia, Indonésia, Irã, Iraque, Irlanda, Islândia, Itália, Jamaica, Japão, Jordânia, Kuwait, Letônia, Líbano, Líbia, Lituânia, Luxemburgo, Malásia, Malawi, Malta, Marrocos, México, Moçambique, Moldávia, Montenegro, Namíbia, Nepal, Nigéria, Noruega, Nova Zelândia, Panamá, Paquistão, Paraguai, Peru, Polônia, Portugal, Catar, Quênia, reino Unido, República Tcheca, República Dominicana, Romênia, Rússia, São Tomé e Príncipe, Senegal, Serra Leoa, Sérvia, Singapura, Síria, Sri Lanka, Suécia, Suíça, Suriname, Tailândia, Taiwan, Tanzânia, Trinidad Tobago, Tunísia, Turquia, Ucrânia, Uruguai, Venezuela, Vietnã, Zâmbia.

Fonte: dados da pesquisa (2020).

Conforme os achados da pesquisa (Tabela 4), quando analisada a amostra total de países (110 países), constata-se uma relação inversa entre os números de casos e a população do país e como a densidade populacional. De acordo com os coeficientes e o nível de significância das regressões a 1\%, as evidências apontam que o índice de contaminação é maior em países com maior PIB per capita $(0,000)$ e há mais casos em países com maior extensão territorial $(0,005)$.

Observa-se na Tabela 4 que há menor ocorrência de mortes onde a população e a densidade populacional são maiores e mais mortes onde o PIB é maior. Já em relação aos casos recuperados, pode-se observar que acontece o inverso: países mais populosos e com maior densidade populacional, tem menos recuperados, mas países com maiores PIB e tamanho demográfico (extensão territorial), possuem maior poder de recuperabilidade.

O índice de contaminação é maior em países com PIB per capita ao nível de $1 \%(0,000)$ e taxa de alfabetização maiores ao nível de $10 \%(0,099)$, a exemplo, países como EUA, Alemanha e França. Inicialmente, pode-se evidenciar tal fatalidade devido ao fato de a doença ter se proliferado em maior número e primeiramente em nações que são grandes centros econômicos mundiais. Nota-se que países com maiores PIB, também possuem grandes populações, e lideram o ranking com maior número de casos e mortes, mas também são os que igualmente conseguem recuperar os pacientes, isso devido ao seu poder econômico.

Países mais desenvolvidos possuem melhores taxas de alfabetização, assim como a população mais alfabetizada também possui um PIB per capita maior, sendo assim, os resultados apontam que países com maior poder econômico foram mais atingidos. Isso não quer dizer que quem está morrendo são as pessoas com maior poder econômico, pelo contrário, pesquisas indicam que os mais pobres estão sendo os mais afetados.

Os achados são suportados pelos estudos do World Bank (2020), constatando-se que os países com maior PIB estão entre os mais afetados pelo vírus e que alguns países, por possuírem maior poder econômico e menor tamanho territorial (Alemanha e Japão), conseguem controlar de melhor forma a doença, achatando a curva do nível de casos (Figura $1)$.

Por sua vez, quando se avalia a extensão territorial (Figura 2), é possível notar a dificuldade que os maiores países possuem em controlar o vírus. O resultado é decorrente do menor controle sobre suas fronteiras, embora sejam nações com alto poder econômico (maiores mercados do mundo). As políticas de isolamento e fechamento do mercado, mesmo que seguissem um mesmo parâmetro mundial, houve uma maior ou menor flexibilidade quanto a elas de país para país, o que acabou causando diferenças nos resultados de minimizar os impactos da proliferação da COVID-19, principalmente em países de grande extensão territorial, como por exemplo, EUA e Brasil. 
Figura 1 - Países com maiores PIB

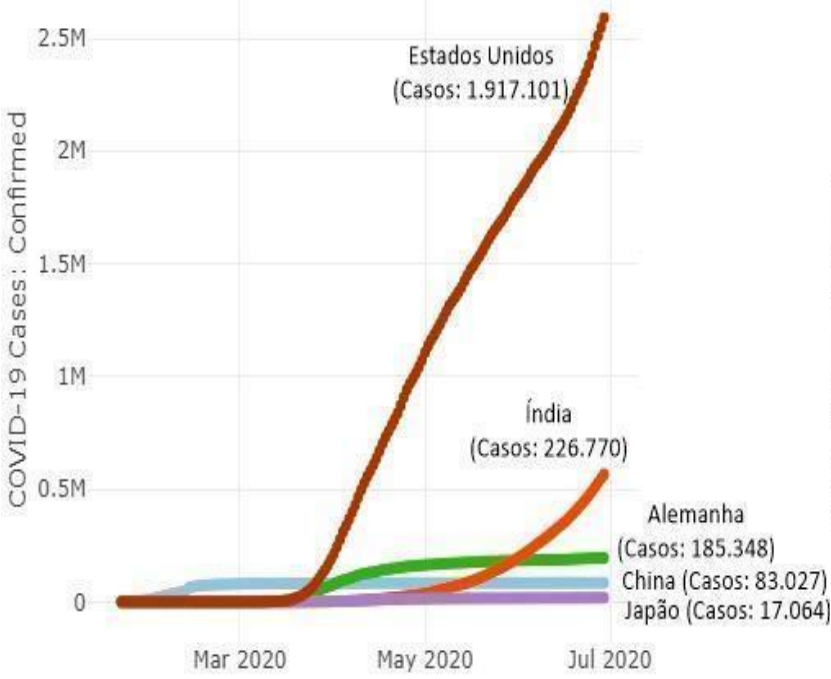

Fonte: World Bank (2020).
Figura 2 - Países de maior extensão territorial

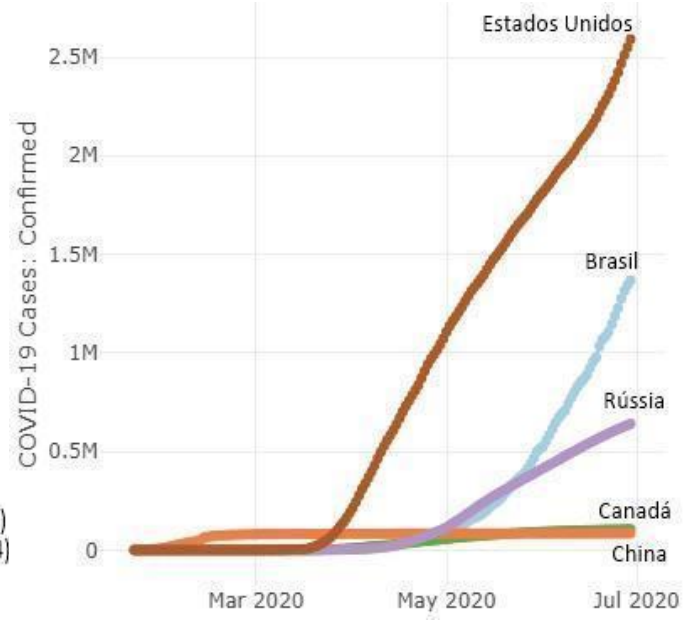

Fonte: World Bank (2020).

Além dos fatores sociais, econômicos e demográficos, outro ponto importante e que pode ser muito significativo em relação a propagação da contaminação é o comportamento da própria população de cada país. Hofstede (2011) identificou que os valores culturais afetam o comportamento e a forma como as pessoas agem habitualmente. Dessa forma, ao inserir as variáveis de cultura no modelo explicativo, comprova-se que o comportamento da população está fortemente associado com a proliferação e mortes causadas pelo vírus (Tabela 5).

Logo, por meio dos dados evidenciados na Tabela 5 é possível elucidar a existência de um maior índice de contaminação em países com maior distância do poder. Existe uma hierarquização social muito forte quando há elevada distância do poder (CHANG, 2003) e aqueles que possuem maior renda não são solidários com os que não possuem, confirmando o cenário de que os pobres são os que mais estão sendo afetados pela COVID-19 (LANCKER; PAROLIN, 2020; SUNMER; HOY; ORTIZ-JUAREZ, 2020). Por sua vez, nos países com menor distância do poder, as pessoas internalizam o compromisso de cooperar e sentir preocupação com o bem-estar de todos (SIEGEL; LICHT; SCHWARTZ, 2011), repercutindo sobre a prevenção da doença.

O estudo também revela a existência de mais casos, mais mortes e maior índice de mortalidade pelo vírus em países com cultura individualista, o que vai ao encontro da pesquisa de Gorodnichenko e Roland (2015), de que os países coletivistas são mais capazes de resolver problemas de ação coletiva, como montar uma resposta coordenada a uma pandemia, enquanto os países com cultura individualista teriam muito mais dificuldade para adotar tal estratégia.

O individualismo indica que as pessoas estão mais preocupadas com o eu, enquanto no coletivismo existe uma maior preocupação com objetivos e metas comuns (TROMPENAARS, 1994). Deste modo, pode-se inferir que na cultura individualista as pessoas não se preocupam com o grupo, tendem a não respeitar as normas sanitárias impostas para a contenção do vírus, e acabam proliferando ainda mais a doença. Logo, o fato de haver significância estatística em relação ao número de recuperados em países com cultura individualista, pode estar atrelado tanto ao fato da recuperabilidade estar intrinsicamente ligada ao número de casos, quanto também pela influência das demais variáveis sociais e econômicas.

Os Estados Unidos pontuam 91\%, em uma escala de 100\% proposto pela Word Bank (2020), e assim tendem a ser uma sociedade altamente individualista. Isso é associado ao 
desejo de expressar as características únicas da identidade pessoal e o foco nas necessidades próprias, em detrimento de olhar para o coletivo. Já a China apresenta um percentual de $20 \%$, indicando ser uma sociedade coletivista, na qual pertencer a um grupo é mais importante. Quando analisado o avanço da doença nestes dois países, é passível compreender quão forte é o impacto desta cultura (Figura 3 e 4). Os Estados Unidos não conseguiram achatar a curva de novos casos, enquanto a China permanece, de certa forma, estabilizada e com número menor de casos.

Ao prosseguir na análise dos dados expostos pela Tabela 5, percebe-se que a regressão aponta que há menos casos, assim como é menor o índice de contaminação em países com orientação a longo prazo. As sociedades com orientação em longo prazo incentivam as pessoas a investirem e a serem econômicos, logo tendem mais facilmente adaptar às tradições aos contextos modernos (HOFSTEDE, 2011). Por sua vez, as sociedades que possuem orientação em curto prazo respeitam as tradições, mas incentivam a gastar e a obter lucros imediatos, além das relações serem vistas como algo importante apenas se for possível tirar proveito delas (HOFSTEDE, 2011). A Coreia do Sul pontua $100 \%$ na escala indicando ser um país de cultura com orientação a longo prazo e possui poucos casos relatados ao longo do tempo, enquanto o Chile pontua $31 \%$, indicando ser um país com cultura mais voltada à orientação de curto prazo e relata um número bem maior de casos e em expressivo crescimento.

Figura 3 - Evolução de Casos Estados Unidos

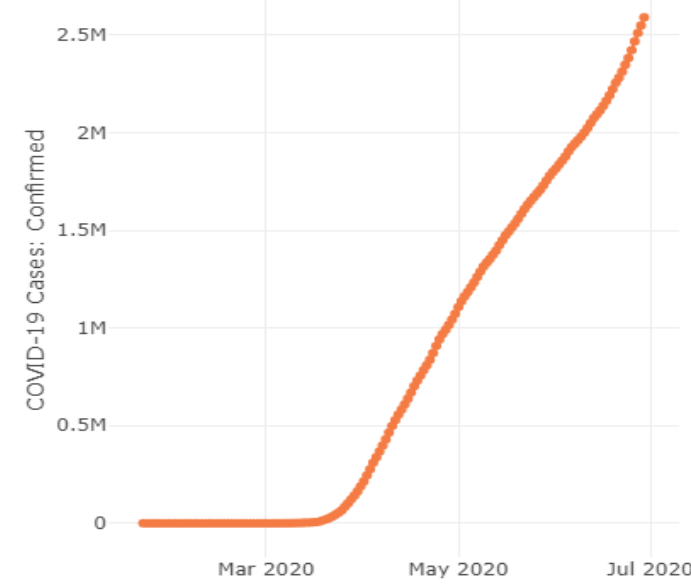

Fonte: World Bank (2020).

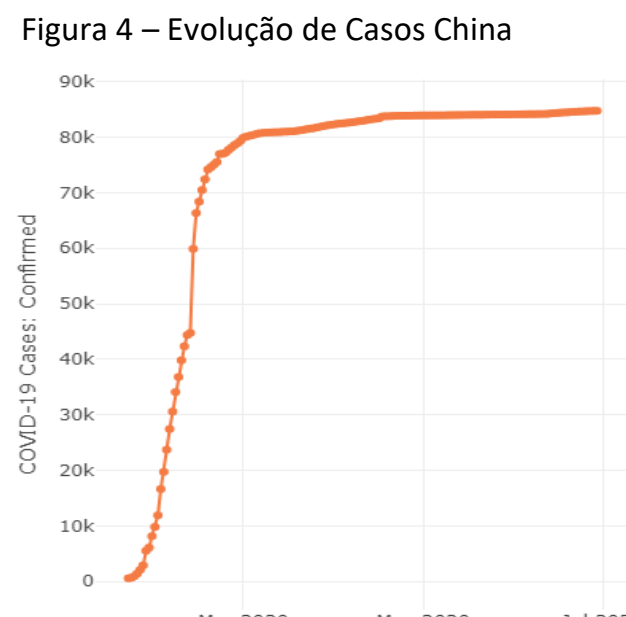

Fonte: World Bank (2020).

É menor o índice de contaminação em países de cultura repreensiva (Tabela 5). As sociedades com alto índice de complacência (alta pontuação) permitem que as pessoas satisfaçam livremente suas necessidades humanas básicas e seus desejos, especialmente aqueles relacionados com o desfrute da vida e o divertimento. Por sua vez, nas sociedades com alto índice de repressão (baixa pontuação) as pessoas suprimem seus impulsos por meio de normas sociais restritivas (HOFSTEDE, 2011). Analisando-se os dados, também se percebe evidências desse traço cultural na propagação da doença, ao se comparar os casos no Paquistão (220,89 milhões de habitantes) e no Brasil (211,48 milhões de habitantes). Enquanto o Paquistão possui cultura de repressão ( $0 \%$ na escala) e apresentava 93.983 casos confirmados, o Brasil com cultura mais complacente (59\% na escala) apresentava 645.771 casos confirmados.

Contatou-se que é maior o índice de mortalidade em países com aversão a incerteza. Em culturas com forte aversão a incerteza, as pessoas tendem a evitar riscos e situações inesperadas e possuem maior preferência para o previsível ou situações controladas, o que 
corrobora os achados de Huynh (2020). Por sua vez, quando a aversão a incerteza é fraca, as pessoas tendem a serem mais tolerantes com aquilo que não podem controlar, sendo que a incerteza é aceita como parte da vida e as pessoas são geralmente mais relaxadas e flexíveis diante de situações desconhecidas (HOFSTEDE, 2011). Grécia, Bélgica e Rússia são exemplos de países com maior aversão a incerteza, enquanto, Hong Kong, Dinamarca e China possuem menos aversão.

Os resultados (Tabela 5) apontam, ainda, que países com maiores índices de percepção de corrupção possuem menos recuperados. O resultado pode indicar que há menos investimento em saúde, resultado da corrupção, que impacta significativamente na qualidade da saúde desses países, corroborando com a pesquisa de Gallego, Prem e Vargas (2020), em que apresentam que os governos são mais propensos a atitudes corruptas, devido aos aumentos dos gastos públicos para combate à doença e pela flexibilização de obtenção de equipamentos. Conforme destacado anteriormente, há pesquisas que indicam que os mais afetados são as classes mais pobres, sendo que estes necessitam prioritariamente do atendimento da saúde pública (LANCKER; PAROLIN, 2020; SUNMER; HOY; ORTIZ-JUAREZ, 2020). Tal resultado, corrobora mais uma vez que os pobres continuam sendo os mais afetados pela COVID-19 e que isso também advém da corrupção do setor público e seus governantes. O Índice de Percepção da Corrupção (IPC) é o principal indicador de corrupção no setor público do mundo, ao ordenar os países de acordo com o grau em que a corrupção é percebida entre os funcionários públicos e políticos.

Tabela 5 - Resultados das regressões com as vinte e cinco nações mais contaminadas

\begin{tabular}{|c|c|c|c|c|c|}
\hline \multirow{3}{*}{$\begin{array}{c}\text { Variáveis } \\
\text { Independentes }\end{array}$} & \multicolumn{5}{|c|}{ Variável dependente } \\
\hline & Confirmados & Mortes & Recuperados & $\begin{array}{c}\text { Índ. de } \\
\text { Contaminação }\end{array}$ & $\begin{array}{c}\text { Índice de } \\
\text { Mortalidade }\end{array}$ \\
\hline & Coeficiente & Coeficiente & Coeficiente & Coeficiente & Coeficiente \\
\hline TXALF & -1.235 .875 & $-49.492,240$ & $-142.925,700$ & $-0,001$ & 0,177 \\
\hline PIBPC & $-10,510$ & $-0,849 * *$ & 0,811 & $2,220 * *$ & $-5,880$ \\
\hline POPUL & 0,000 & $-0,000 *$ & 0,000 & 5,800 & $-4,900$ \\
\hline $\mathrm{IDH}$ & 334,590 & 18,496 & 175,699 & 0,000 & 0,000 \\
\hline PIB & $0,059 * *$ & $0,003^{*}$ & 0,007 & $-1,170$ & $-2,470$ \\
\hline TAMKM & $0,057 * *$ & $0,002 * * *$ & 0,013 & 2,540 & $-1,950$ \\
\hline DENSPOP & -3.052 .595 & $-173.075,600 * *$ & $-750.079,400$ & $-0,016$ & $-0,121$ \\
\hline DESEMP & $-2.323,388$ & 78,334 & $5.648,785$ & 0,000 & 0,000 \\
\hline DIVPUB & 322,168 & $284,590 * * *$ & $-402,801$ & 0,000 & 0,001 \\
\hline PERCCOR & $8.524,501$ & 523,411 & $-1.804,636$ & 0,000 & 0,000 \\
\hline DISTPODER & $-423,786$ & 247,051 & $-1.455,330$ & 0,000 & 0,001 \\
\hline INDIVID & $11.215,140 * *$ & $622,637 * *$ & $1.851,970$ & 0,000 & 0,000 \\
\hline MASCUL & $-4.377,546$ & $-239,349$ & 131,685 & 0,000 & 0,000 \\
\hline AVINC & $7.173,749$ & 46,326 & $2.479,227$ & 0,000 & $-0,001$ \\
\hline ORILPRAZO & $-9.507,833 * *$ & $-404,691 * *$ & $-1.118,906$ & $-0,000 * *$ & 0,000 \\
\hline INDULG & $-1.021,163$ & 48,584 & $-456,326$ & 0,000 & 0,000 \\
\hline Constante & $463.474,700$ & $-3.336,650$ & $9.255,971$ & 0,000 & $-0,110$ \\
\hline Estatística F & 4,55 & 8,36 & 2,68 & 2,84 & 1,29 \\
\hline $\mathrm{R}^{2}$ & 0,9009 & 0,9435 & 0,8426 & 0,8505 & 0,7207 \\
\hline VIF & 6,5 & 6,5 & 6,5 & 6,5 & 6,5 \\
\hline DW & 1,60 & 1,95 & 1,94 & 1,87 & 1,88 \\
\hline $\mathrm{N}$ & 25 & 25 & 25 & 25 & 25 \\
\hline
\end{tabular}

Legenda: * significativo a $1 \% ;{ }^{* *}$ significativo a $5 \% ;{ }^{* * *}$ Significativo a $10 *$.

Amostra: Estados Unidos, Brasil, Rússia, Reino Unido, Espanha, Itália, Índia, Alemanha, Peru, Turquia, Irã, França, Chile, México, Arábia Saudita, Canadá, Paquistão, China, Catar, Bangladesh, Bélgica, Holanda, Biolorússia, Suécia e Equador.

Fonte: dados da pesquisa (2020).

Complementarmente, o estudo também considerou uma amostra com os 25 países mais afetados pela COVID-19, com os resultados apresentados na Tabela 5 . No conjunto, os 
25 países mais afetados somam $90,06 \%$ dos casos confirmados no mundo e $95,64 \%$ de todas as mortes ocorridas pela COVID-19 (Tabela 1), são economicamente mais ativos e, consequentemente, os grandes centros de cada país acabaram por ser as cidades mais afetadas.

Devido a expressividade dos casos destas 25 nações, surge a oportunidade de explorar como as variáveis sociais, demográficas, econômicas e culturais se comportam nesse grupo, isoladamente (Tabela 5). O estudo confirma que fatores institucionais e culturais se combinam para explicar parcialmente os resultados das taxas de crescimento e mortalidade do COVID19 (GELFAND et al., 2020).

Constatou-se que nos 25 países mais contaminados, os casos e o número de mortes são maiores em países territorialmente maiores e onde há maior PIB. O exemplo mais cabível é os Estados Unidos, que é um grande país em extensão territorial, possui o 20 maior PIB do mundo e está enfrentando muita dificuldade para controlar a disseminação do vírus. Os resultados ainda apontam que há uma relação inversa entre o número de casos, a cultura individualista e orientação a longo prazo.

Quando observadas as 25 maiores nações do mundo temos uma relação positiva entre os números da COVID-19 e a cultura individualista, assim como na análise com os 110 países. Países como Estados Unidos, Brasil, Rússia, Reino Unido, Espanha, Itália, Índia e Alemanha, são considerados individualistas e da mesma forma estão entre aqueles que lideram o ranking em número de casos e mortes pelo coronavírus no mundo.

Em relação a mortalidade, identificou-se uma relação inversamente proporcional ao PIB per capita, tamanho da população, densidade populacional e orientação a longo prazo, e uma relação diretamente proporcional com o PIB do país, o tamanho territorial, a dívida pública (EUA, Brasil, Rússia, Reino Unido e Espanha possuem dívidas públicas superiores a $100 \%$ do seu PIB) e a cultura individualista.

O índice de contaminação é maior em países onde há uma população mais rica (maior PIB per capita) e menor onde há uma cultura de orientação a longo prazo. As mortes e o índice de mortalidade podem variar em cada país, de acordo com as suas políticas de contenção, isolamento e quarentena, assim como as diferenças nas condições de saúde subjacentes e na capacidade do sistema de saúde (WALKER et al., 2020). Cabe salientar que, segundo o estudo do Grupo de Resposta ao novo coronavírus do Imperial College London, a ausência de intervenções resultaria em mais de 7 bilhões de infecções e mais de 40 milhões de mortes em todo o mundo, ainda em 2020 (WALKER et al., 2020; ELSLAND; O'HARE, 2020).

Os resultados confirmam que a disseminação do COVID-19 representa uma crise global de saúde pública, mas algumas nações foram mais eficazes do que outras em limitar a disseminação do vírus durante os estágios iniciais da pandemia (GELFAND et al., 2020).

\section{CONSIDERAÇÕES FINAIS}

O estudo teve por objetivo analisar os fatores sociais, econômicos, culturais e demográficos que influenciam na propagação dos casos da COVID-19 e na mortalidade das pessoas em âmbito internacional. Os resultados sugerem que as variáveis determinantes da COVID-19 podem ser explicadas tanto por fatores sociais, econômicos e demográficos quanto pelo comportamento característico da cultura de cada nação.

O estudo fornece contribuição teórica ao avaliar dados de 110 nações e demonstrar que as dimensões culturais são relevantes para explicar os resultados. Nações com cultura mais individualista, com maior distância do poder e com maior aversão à incerteza mostraram maiores índices de mortalidade. Por sua vez, países com orientação de curto prazo e mais 
complacentes, apresentaram maiores índices de contaminação. Em relação ao número de casos, nações com menores populações e menor densidade populacional, com maior extensão territorial e maior riqueza interna mostraram-se mais propensas a apresentar contaminados.

A contribuição prática consiste nas evidências de que os fatores culturais e o poder socioeconômico podem estar atrelados com a adoção de políticas para contenção de novas pandemias ou epidemias. Cada país possui suas características sociais, demográficas, econômicas e culturais que induzem as estratégias de enfrentamento às crises, no entanto observar o contexto das variáveis culturais pode contribuir na evidenciação de estratégias mais eficazes, especialmente diante de adversidades como no caso de uma pandemia. $O$ estudo também permite observar que o contexto político das nações e a forma de enfrentar o vírus refletiu nos dados de contaminados pelo COVID-19.

Os dados referentes ao vírus foram coletados no dia 05 de junho de 2020, quando o número de casos totais no mundo eram de 6.416 .828 . Frente a tal perspectiva, pode-se elencar a evolução constante do cenário pandêmico, como uma limitação da pesquisa, pois os resultados evidenciados têm poder de representar apenas os reflexos inferidos até o período de coleta da amostra. Outro aspecto que pode se destacar como limitação é em relação aos testes de detecção do vírus, que podem não estar ocorrendo ou quando ocorrem demoram para compor as bases de dados. Também não foram objeto deste estudo, as medidas governamentais utilizadas para a contenção do vírus e como tais medidas podem surtir efeito na amostra.

Para estudos futuros recomenda-se continuar a testar a influência das variáveis sociais, econômicas, demográficas e culturais conforme a evolução dos casos, a fim de verificar se a relação evidenciada nos resultados desta pesquisa, permanecerá futuramente em estágios mais avançados da pandemia. Ainda, sugere-se a análise das taxas de mortalidade de cada país relacionadas aos aspectos de políticas de contenção, isolamento ou quarentena, bem como na capacidade de absorção do sistema de saúde de cada nação.

\section{AGRADECIMENTOS}

Os autores agradecem a Fundação de Amparo à Pesquisa e Inovação do Estado de Santa Catarina (FAPESC), pelo apoio financeiro à pesquisa.

\section{REFERÊNCIAS}

ANDERSON, R. M.; HEESTERBEEK, H.; KLINKENBERG; D.; HOLLINGSWORTH, T. D. How will country-based mitigation measures influence the course of the COVID-19 epidemic? The Lancet, v. 395, n. 10228, p. 931-934, 2020. DOI: 10.1016/S0140-6736(20)30567-5.

AVENI, A. Estratégias pelo trabalho no futuro devido a pandemia Covid-19. Revista Processus de Políticas Públicas e Desenvolvimento Social, Águas Claras, v. 2, n. 3, p. 04-14, 2020. Disponível em: http://periodicos.processus.com.br/index.php/ppds/article/view/187. Acesso em: 20 jun. 2020.

BHUSAL, M. K. The World After COVID-19: An opportunity for a new beginning. International Journal of Scientific and Research Publications, Helsinki, v. 10, n. 5, p. 735-741, 2020. DOI: 10.29322/IJSRP.10.05.2020. p10185. 
BRESSER-PEREIRA, L. C. Financiamento da Covid-19, inflação e restrição fiscal. Brazilian Journal of Political Economy, São Paulo, v. 40, n. 4, p. 604-621, 2020. DOI: https://doi.org/10.1590/0101-31572020-3193

CECCHETTI, S.; MOHANTY, M.; ZAMPOLLI, F. The real effects of debt. BIS Working Papers, Aeschenplatz, n. 352, 2011.

CHANG, L. C. An examination of cross-cultural negotiation: Using Hofstede framework. Journal of American Academy of Business, Nova lorque, v. 2, n. 2, p. 567-570, 2003.

CIA - CENTRAL INTELLIGENCE AGENCY. The Word Factbook. Langley, 2020. Disponível em: https://www.cia.gov/library/publications/the-world-factbook/rankorder/2147rank.html. Acesso em: 20 jun. 2020.

CEPAL - COMISSÃO ECONÔMICA PARA A AMÉRICA LATINA E O CARIBE. América Latina y el Caribe ante la pandemia del COVID-19: efectos económicos y sociales. Santiago, 2020. Disponível em: https://repositorio.cepal.org/bitstream/handle/11362/45337/6/ S2000264_es.pdf. Acesso em: 06 jun. 2020.

ELSLAND, S. L. V., O'HARE, R. Coronavirus pandemic could have caused $\mathbf{4 0}$ million deaths if left unchecked. Londres, 2020. Disponível em: https://www.imperial.ac.uk/news/196496/coronavirus-pandemic-could-have-caused-40/. Acesso em: 30 jun. 2020.

FEREIRA JUNIOR, R. R.; RITA, L. P. S. Impactos da Covid-19 na Economia: limites, desafios e políticas. Cadernos de Prospecção, Salvador v. 13, n. 2, p. 459-476, 2010. DOI: http://dx.doi.org/10.9771/rf.v1i7.37324.

FREY, C. B.; CHEN, C.; PRESIDENTE, G. Democracy, Culture, and Contagion: Political Regimes and Countries Responsiveness to Covid-19. Covid Economics, Reino Unido, v. 18, p. 1-20, 2020. Disponível em: https://www.oxfordmartin.ox.ac.uk/downloads/academic/DemocracyCulture-and-Contagion_May13.pdf. Acesso em: 20 jun. 2020.

FREITAS, C. M.; SILVA, I. V. M.; CIDADE, N. C. COVID-19 AS A GLOBAL DISASTER: Challenges to risk governance and social vulnerability in Brazil. Ambiente e sociedade, São Paulo, v. 23, 2020. DOI: https://doi.org/10.1590/1809-4422asoc20200115vu2020l3id.

FURTADO, C. Desenvolvimento e subdesenvolvimento. Rio de Janeiro: Centro Celso Furtado. Contraponto, 2009.

GALLEGO, J. A.; PREM, M.; VARGAS, J. F. Corruption in the Times of Pandemia. Working paper, Bogotá, n. 44, 2020. DOI: http://dx.doi.org/10.2139/ssrn.3600572.

GAMA NETO, R. B. Impactos da Covid-19 sobre a economia mundial. Boletim de Conjuntura (BOCA), Boa Vista, v. 2, n. 5, p. 113-127, 2020. DOI:

http://dx.doi.org/10.5281/zenodo.3786698. 
GELFAND, M.; JACKSON, J. C.; PAN, X.; NAU, D.; DAGHER, M. M.; VAN LANGE, P.; CHIU, $C$. The importance of cultural tightness and government efficiency for understanding COVID19 growth and death rates. Psyarxiv, [S. I.], 2020. DOI: 10.31234/osf. io/m7f8a.

GORODNICHENKO, Y.; ROLAND, G. Culture, institutions, and the wealth of nations. Review of Economics and Statistics, Londres, v. 99, n. 3, p. 402-416, 2017. DOI: 10.1162/REST_a_00599

HOFSTEDE, G. Dimensionalizing cultures: The Hofstede model in context. Online readings in psychology and culture, Reino Unido, v. 2, n. 1, p. 1-26, 2011. DOI: 10.9707/23070919.1014.

HOFSTEDE INSIGHTS. Compare countries. Mikonkatu, 2020. Disponível em: https://www.hofstede-insights.com/. Acesso em: 25 maio 2020.

HUYNH, T. L. D. Does culture matter social distancing under the COVID-19 pandemic? Safety Science, Netherlands, v. 130, p. 1-19, 2020. DOI: https://doi.org/10.1016/j.ssci.2020.104872.

HU, M., ROBERTS, J., AZEVEDO, G. P., MILNER, D. The role of built and social environmental factors in Covid-19 transmission: A look at America's capital city. Sustainable Cities and Society, p. 102580, 2020. DOI: https://doi.org/10.1016/j.scs.2020.102580.

INTERNATIONAL MONETARY FUND. Gross domestic product, current prices. Washington, dez., 2019. Disponível em: https://ec.europa.eu/eurostat/web/products-datasets//tec00001. Acesso em: 20 mai. 2020.

KRAEMER, M. U.; YANG, C-H.; GUTIERREZ, B.; WU; C-H.; KLEIN, B.; PIGOTT, D. M. The effect of human mobility and control measures on the COVID-19 epidemic in China. Science, Washington, v. 368, n. 6490, p. 493-497, 2020. DOI: 10.1126/science.abb4218.

KRASTEV, T. V.; PETROV, G. M.; MARINOV, B. S. Increasing the Risk of Corruption Activities during a COVID-19 Pandemic, International Scientific Journal, Internauka, Ucrânia, v. 92, n. 12, 2020. DOI: http://dx.doi.org/10.2139/ssrn.3674427.

KUMAR, M.; WOO, J. Public debt and growth. IMF working papers: International Monetary Fund. Washington, n. 10. v. 174, p. 1-47, 2010.

LAING, T. The economic impact of the Coronavirus 2019 (Covid-2019): Implications for the mining industry. The Extractive Industries and Society, Países Baixos, v. 7, n. 2, p. 580-582, 2020. DOI: 10.1016/j.exis.2020.04.003.

LANCKER, W. V; PAROLIN, Z. COVID-19, school closures, and child poverty: a social crisis in the making. The Lancet Public Health, Nova lorque, n. 5, p. 243-244, 2020. DOI: 10.1016/S2468-2667(20)30084-0.

LEMOS, P.; ALMEIDA-FILHO, N.; FIRMO, J. COVID-19, desastre do sistema de saúde no presente e tragédia da economia em um futuro bem próximo. Brazilian Journal of 
Dyeniffer Packer Eigenstuhler, Francieli Pacassa, Silvana Dalmutt Kruger e Sady Mazzioni

Implantology and Health Sciences, Macapá, v. 2, n. 4, p. 39-50, 2020. DOI: 10.36557/26748169.2020v2n4p39-50.

MARANHÃO, R., A.; SENHORAS, E. M. Pacote econômico governamental e o papel do BNDES na guerra contra o novo coronavírus. Boletim de Conjuntura (BOCA), Boa Vista, v. 2, n. 4, p. 27-39, 2020. DOI: http://dx.doi.org/10.5281/zenodo.3748888.

MORROW, G.; ROTHWELL, C.; BURFORD, B.; ILLING, J. Cultural dimensions in the transition of overseas medical graduates to the UK workplace. Medical Teacher, Reino Unido, v. 35, $\mathrm{n}$. 10, p. 1537-1545, 2013. DOI: 10.3109/0142159X.2013.802298.

MURSHED, S. M. Capitalism and COVID-19: Crisis at the Crossroads. Peace Economics, Peace Science and Public Policy, Alemanha, v. 1, p. 1-8, 2020. DOI: 10.1515/peps-2020-0026.

NAÇÕES UNIDAS BRASIL. OIT: quase 25 milhões de empregos podem ser perdidos no mundo devido à COVID-19. Brasília, 2020. Disponível em:

https://www.ilo.org/brasilia/noticias/WCMS_738780/lang--

pt/index.htm\#: :text=GENEBRA\%20(OIT\%20Not\%C3\%ADcias)\%20\%E2\%80\%93A,Internacion al\%20do\%20Trabalho\%20(OIT). Acesso em: 20 jun. 2020.

NICOLA, M., ALSAFI, Z.; SOHRABI, C.; KERWAN, A.; AL-JABIR, A.; IOSIFIDIS, C.; AGHA, M.; AGHA, R. The socio-economic implications of the coronavirus pandemic (COVID-19): A review. International Journal of Surgery, Londres, v. 78, p. 185, 2020. DOI:

10.1016/j.ijsu.2020.04.018.

OMS - ORGANIZAÇÃO MUNDIAL DA SAÚDE. Coronavirus disease (COVID-2019) situation reports. Genebra, 2020. Disponível em: https://www.who.int. Acesso em: 26 jun. 2020

OPAS - ORGANIZAÇÃO PAN-AMERICANA DA SAÚDE. Folha informativa - COVID-19 (doença causada pelo novo coronavírus). Washington, jun., 2020. Disponível em: https://www.paho.org. Acesso em: 27 jun. 2020

PIKETTY, T. O capital no século XXI. Rio de Janeiro: Intrínseca, 2014.

PNUD - PROGRAMA DAS NAÇÕES UNIDAS PARA O DESENVOLVIMENTO. Ranking IDH Global 2014. Nova lorque, 2020 Disponível em:

https://www.br.undp.org/190ontente/brazil/pt/home/idh0/rankings/idh-global.html. Acesso em 20 mai. 2020.

REINHART, C. M.; ROGOFF, K. S. Growth in a time of debt. American Economic Review, Pittsburgh, v. 100, n. 2, p. 573-78, 2010. DOI: https://doi.org/10.3386/w15639.

SIEGEL, J. I.; LICHT, A. N.; SCHWARTZ, S. H. Egalitarianism and international investment. Journal of Financial Economics, Nova lorque, v. 102, n. 3, p. 621-642, 2011. DOI: 10.1016/j.jfineco.2011.05.010. 
SOUZA, D. O. The COVID-19 pandemic beyond Health Sciences: reflections on its social determination. Ciência \& Saúde Coletiva, Rio de Janeiro, v. 25, p. 2469-2477, 2020. DOI: 10.1590/1413-81232020256.1.11532020.

THE WORLD BANK. Understanding the Coronavirus (COVID-19) pandemic through data. Washington, jun., 2020. Disponível em: http://datatopics.worldbank.org/universal-healthcoverage/coronavirus/. Acesso em 20 jun. 2020.

TRADING ECONOMICS. Taxa de Desemprego - Lista de Países. Nova lorque, 2020.

Disponível em: https://pt.tradingeconomics.com/country-list/unemployment-rate. Acesso em 20 mai. 2020.

TRANSPARÊNCIA INTERNACIONAL BRASIL. Índice de Percepção da Corrupção 2019. São Paulo, 2019. Disponível em: https://transparenciainternacional.org.br/ipc/. Acesso em 20 mai. 2020.

TROMPENAARS, F. Nas ondas da cultura: como entender a diversidade cultural nos negócios. São Paulo: Educator, p. 13-28, 1994.

WALKER. P. GT. et al. The global impact of COVID-19 and strategies for mitigation and suppression. WHO Collaborating Centre for Infectious Disease Modelling, MRC Centre for Global Infectious Disease Analysis, Abdul Latif Jameel Institute for Disease and Emergency Analytics, Imperial College London, p. 1-19, 2020. DOI: https://doi.org/10.25561/77735. 